\title{
Alendronate Alleviated Femoral Head Necrosis and Upregulated BMP2/EIF2AK3/EIF2A/ATF4 Pathway in Liquid Nitrogen Treated Rats
}

\author{
Ke Rong' \\ Xiaoliu $\mathrm{Li}^{2}$ \\ Weimin Jiang $\mathbb{D}^{\prime}$ \\ Xuhua $\mathrm{Wu}^{\prime}$ \\ Qingquan $\mathrm{Xia}^{2}$ \\ Jie Chen' \\ Xiaofan Yin ${ }^{2}$ \\ 'Department of Orthopedics, The First \\ Affiliated Hospital of Soochow University, \\ Soochow, 215006, People's Republic of \\ China; ${ }^{2}$ Department of Orthopedics, \\ Minhang Hospital, Fudan University, \\ Shanghai, 20I199, People's Republic of \\ China
}

Background: Osteonecrosis of the femoral head (ONFH) seriously affects the quality of life and labor ability of patients. It is urgent and vital to find the methods for necrosis clinical treatment.

Objective: This study aims to study the potential protective effects of Alendronate in the early stage of femur head necrosis.

Methods: Ten clinal ONFH tissue samples were employed. H\&E staining was employed for the observation of the pathological characteristics of ONFH. The rat model $(n=12)$ was established by the treatment of liquid nitrogen and then treated with Alendronate. The protein expression of BMP2, EIF2AK3, EIF2A and ATF4 were detected via Western blotting and IHC.

Results: Fibrin and necrotizing granulation tissue were observed in ONFH tissues with lymphocytes and plasma cells infiltrating in the necrotic area, exhibiting the inflammatory muscle with abnormal shape and color. In the Model group, the BMP2 and ATF4 were mainly distributed in the cell boundaries. The relative protein expression of BMP2, EIF2AK3, EIF2A, ATF4 was decreased in the Model group, compared to the NC group, which was partially recovered by the Alendronate application.

Conclusion: Alendronate application partially reversed the suppression of expression of BMP2, EIF2AK3, EIF2A, ATF4 caused by liquid nitrogen. Alendronate could be a promising strategy of curing ONFH via targeting BMP2/EIF2AK3/EIF2A/ATF4 pathway.

Keywords: femoral head necrosis, alendronate, BMP2, ATF4

\section{Introduction}

Osteonecrosis of the femoral head $(\mathrm{ONFH})$ is the death of osteocytes, fracture of trabecula and collapse of bone tissue due to lack of blood supply which commonly affects femur. ${ }^{1-3}$ In the early stage of the disease, its pathological manifestations are dull pain and hidden pain of the hip joint, accompanied by the aggravation of hip pain after exercise, as well as limitation of motion and claudication in severe cases. Without timely treatment, the necrotic area will seriously affect the quality life of patients, and posing a heavy burden on society and families. ${ }^{4,5}$ In consideration of the consequences incurred by ONFH, it is urgent and vital to find the necrosis mechanism.

Bone morphogenetic protein 2 (BMP2) exists in the bone matrix, which can induce the differentiation of stromal cells into chondrocytes and osteocytes. ${ }^{6,7}$ Recently, transmembrane protein 119 (TMEM119) has been identified as an 
osteoinductive factor, which promotes the differentiation of myoblasts into osteoblasts by inducing BMP2 levels. ${ }^{8}$ At the same time, TMEM119 has a signal peptide of 18 amino acids, which directs the nascent polypeptide to the endoplasmic reticulum. ${ }^{7,9}$ One of the key endoplasmic reticulum stress systems is the EIF2AK3-EIF2A-ATF4 signaling pathway. Therefore, strengthening the function of those proteins can further suppress the structural changes in femoral bone tissue. Alendronate, a nitrogencontaining bisphosphonate, is generally considered the effective drug for the treatment of osteoporosis, due to the evidence of its great potential to inhibit bone resorption and increase bone mineral destiny. ${ }^{10,11}$ Thus, Alendronate might contribute to controlling bone turnover and preventing the progression of ONFH and it is worth further studying the molecular mechanism using Alendronate for treatment in the early stage of femur head necrosis.

In this paper, clinical samples of femoral head necrosis were collected to detect the expression levels of BMP2, AFT4, TMEM119. And the rat model of ONFH was established via liquid nitrogen to observe the effect of Alendronate on ONFH in rats and explore its mechanism of action to provide an experimental basis for the application of Alendronate in the treatment of ONFH.

\section{Methods}

\section{Patients and Tissue Samples}

ONFH tissue samples were obtained from The First Affiliated Hospital of Soochow University (Suzhou, China). Patients with total hip arthroplasty due to femoral neck fracture or ONFH were included, while those under the age of 18 or those with missing baseline information were excluded from this study. The surgical resected ONFH tissues were provided by 10 patients $(56.4 \pm 4.2$ years old, 4 males and 6 females) that were collected during total hip arthroplasty at The First Affiliated Hospital of Soochow University, with 10 normal tissues (52.5 \pm 5.6 years old, 5 males and 5 females) selected as the control. The tissues were taken by chiseling along the longitudinal line of the femoral head and stored at $-80^{\circ}$ C. ${ }^{12}$ This experiment was approved by the institutional ethical board of The First Affiliated Hospital of Soochow University (Suzhou, China) and conducted in accordance with the Declaration of Helsinki. Informed consents were signed by all donors according to the International Ethical Guidelines.

\section{Histological Staining}

The ONFH tissues and normal tissues were primarily fixed with $4 \%$ paraformaldehyde at $4{ }^{\circ} \mathrm{C}$ overnight. ${ }^{13}$ Then, the samples were cut into slices $5 \mu \mathrm{m}$ and underwent Hematoxylin-eosin (H\&E) staining and Masson's staining (Solarbio, Beijing, China) as per the guideline of manufacturers. After that, slices were blocked with neutral resins and the images of tissues were taken by a light microscope (Olympus, Tokyo, Japan).

\section{Animal Experiments}

All animal experiments were performed in accordance with the National Research Council Guide for Care and Use of Laboratory Animals and the Declaration of The First Affiliated Hospital of Soochow University and approved by the Animal Research Ethics Committee of The First Affiliated Hospital of Soochow University, Suzhou, China. A total of 36 Sprague-Dawley (SD) rats (8-12 weeks old) were purchased from Shrek laboratory animal Co., Ltd (Shanghai, China) and kept in a clean and sterile laboratory with constant temperature at $25^{\circ} \mathrm{C}$ for and constant humidity at $50 \%$. All the rats were free to the food and water with $12 \mathrm{~h}$ light cycle per day. The tested animals were divided into three groups (12 rats per group): NC group, Model group and Alendronate group. The Model group and Alendronate group were frozen with liquid nitrogen to establish the femoral head necrosis model while the NC group was served as the normal control. After that, the Alendronate group was treated with $100 \mu \mathrm{M}$ Alendronate for 10 days. Then, the rats were sacrificed by $\mathrm{CO}_{2}$ and isoflurane anesthesia, and the femoral head samples were collected and imaged.

\section{Western Blotting Analysis}

Briefly, the bone tissue was transferred into a mortar filled with liquid nitrogen and ground into powder. Then the powdered bone tissue was collected into a $1.5 \mathrm{~mL}$ EP tube and added with protein lysate, which was lysed at $0^{\circ} \mathrm{C}$ for $30 \mathrm{~min}$. Then the samples were centrifuged at $4^{\circ} \mathrm{C}$ for $15 \mathrm{~min}$ at $12,000 \mathrm{rpm}$. The supernatant containing total protein was extracted and stored at $-80^{\circ} \mathrm{C}$. The total protein was dissolved with trypsin and quantified using a BCA Kit (Beyotime, Haimen, China). The protein samples were then applied to a $10 \%$ polyacrylamide gel for separation, and proteins were subsequently subjected onto polyvinylidene fluoride membranes (Thermo Fisher, CA, USA), followed by incubation with primary antibodies 
purchased from Abcam (Cambridge, MA, USA) including anti-human BMP2 (ab14933, 1:1000), anti-human ATF4 (ab184909, 1:5000), anti-human TMEM119 (ab210405, 1:1000), anti-rat BMP2 (ab214821, 1:1000), anti-rat ATF4 (ab186284, 1:500), anti-rat EIF2AK3 (ab229912, $1: 1000)$, anti-rat EIF2A (ab169528, 1:1000) and anti- $\beta$ actin (ab8226, 1:2000) antibodies. The membranes were then incubated with the secondary antibody goat anti-rat IgG (ab150165, 1:2000) for $1 \mathrm{~h}$ at room temperature. ${ }^{14}$ The density of blots was normalized by using $\beta$-actin as an internal reference, and captured by ImageJ software (Rawak Software Inc., Stuttgart, Germany).

\section{Immunohistochemistry (IHC)}

The IHC assay was conducted as per the standard protocol published elsewhere. ${ }^{15}$ The ONFH tissues in each group were prepared into paraffin tissue sections of about $5 \mu \mathrm{m}$, followed by a series of instructions including dewaxing, hydration, and blocking. The primary antibodies anti-rat
BMP2 (1:500) and anti-rat ATF4 (1:1000) were added and incubated at $37^{\circ} \mathrm{C}$ for $1 \mathrm{~h}$. After that, secondary antibody Rabbit anti-rat IgG (1:1000) was blocked for $20 \mathrm{~min}$. The protein expression in each tissue was observed by a fluorescence microscope (Olympus, Tokyo, Japan). The histochemical score was calculated as the product of the positive staining percentage score and the staining intensity score using ImageJ software.

\section{Results}

The Pathological Characteristics of Osteonecrosis of the Femoral Head

As shown in Figure 1, no cystic cavity in the central part of femoral head necrosis was observed in ONFH tissues obtained from clinical samples, compared to the normal tissues. Also, fibrin and necrotizing granulation tissue were captured with lymphocytes and plasma cells infiltrating in the necrotic area.
A

Osteonecrosis tissues

Normal tissues

B

\section{Osteonecrosis} tissues
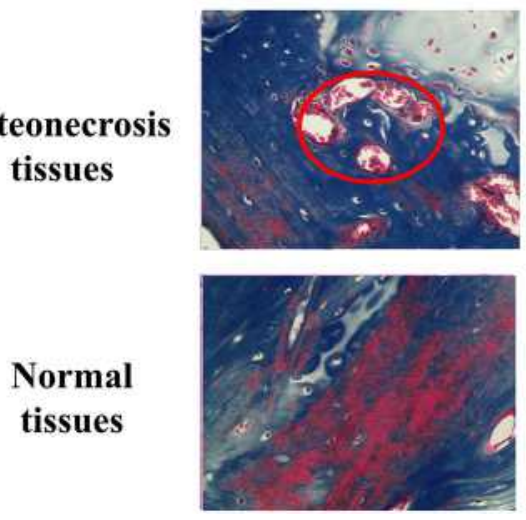

Case 1

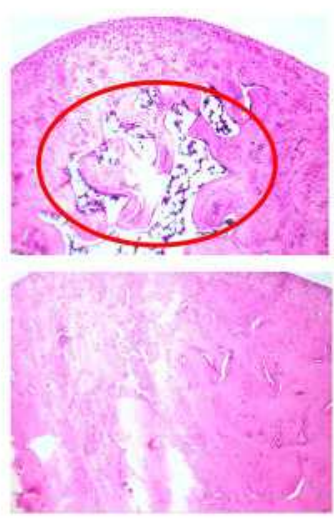

Case 1
Case 2

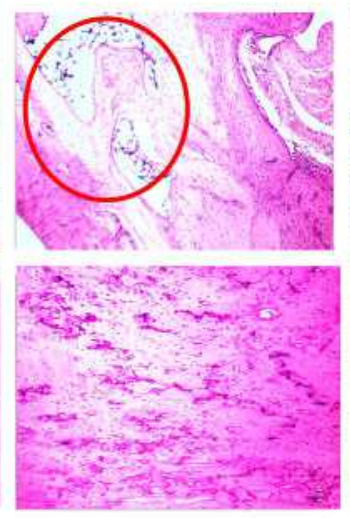

Case 2
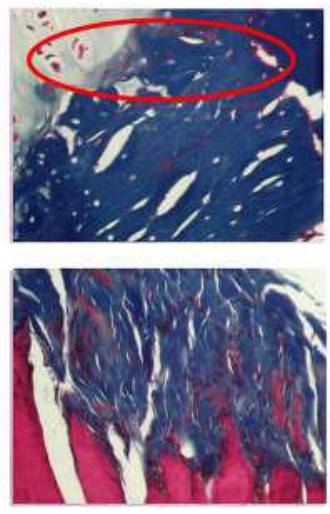

Case 3

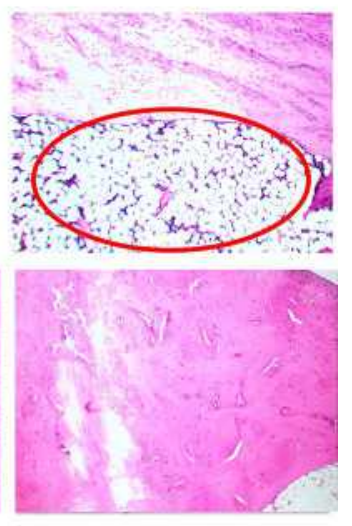

Case 3
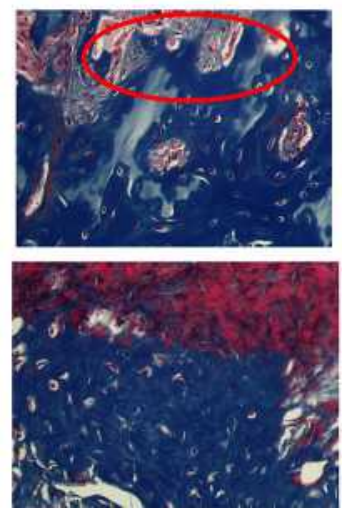

Figure I The pathological characteristics of osteonecrosis of the femoral head. (A) H\&E staining. (B) Masson's staining. Three cases represented samples selected randomly from three individuals. Scale bar $=50 \mu \mathrm{m}$. 


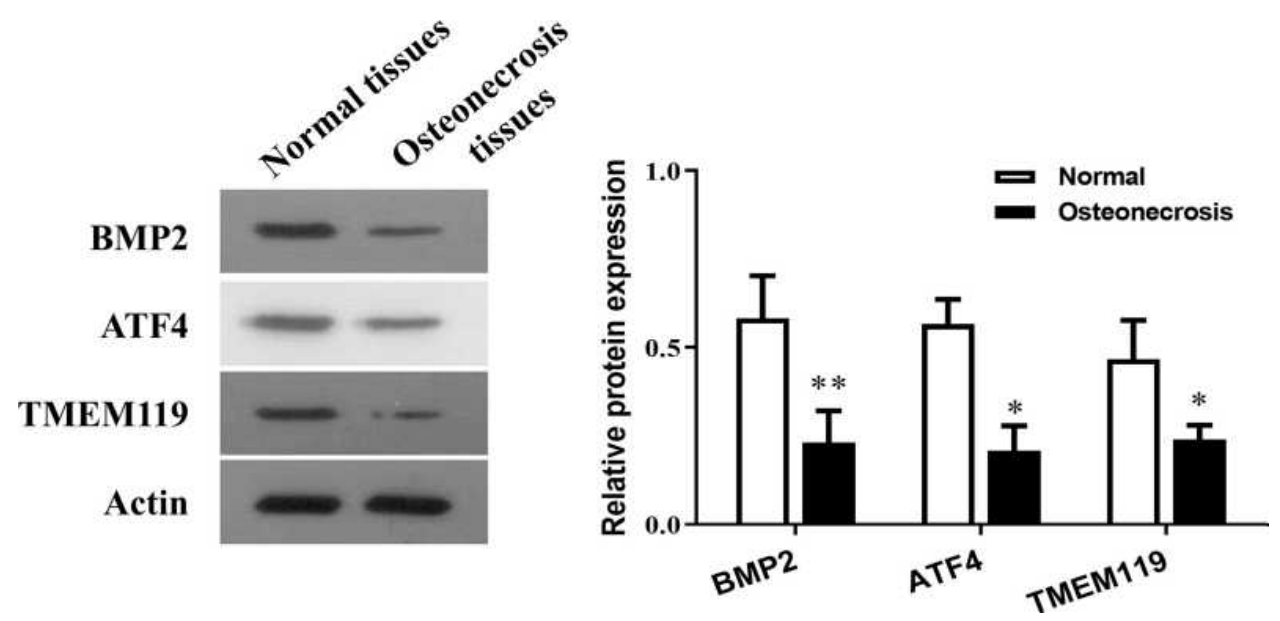

Figure 2 The relative protein expression of BMP2, ATF4 and TMEMII 9 in normal and osteonecrosis tissue detected by Western blotting. * $<<0.05$, **p<0.0I compared with normal tissues.

\section{The Protein Expression of BMP2, ATF4} and TMEMII9 Was Reduced in

\section{Osteonecrosis Tissues}

As shown in Figure 2, the relative protein expression of related signaling pathway in normal and osteonecrosis tissue obtained from clinical samples was detected using Western blotting. The protein expression of BMP2 $(p<0.01)$ ATF4 $(p<0.05)$ and TMEM119 $(p<0.05)$ was all significantly reduced in osteonecrosis tissues, compared with those in normal tissues.

\section{Alendronate Alleviated Osteonecrosis of} Femoral Head Caused by Liquid Nitrogen Seen from Figure 3, the femoral head in the Model group exhibited severer necrosis than that in the NC group, as the inflammatory muscle presented abnormal shape and color.
The Alendronate reversed osteonecrosis of femoral head caused by liquid nitrogen with relatively normal shape, but still exerted necrosis characteristics.

\section{Alendronate Reversed the Suppression of Protein Expression of BMP2/ATF4 \\ Pathway in Osteonecrosis Tissues}

The IHC assay presented the reduced expression of BMP2 (Figure 4A) and ATF4 (Figure 4B) in osteonecrosis tissues, compared to the NC group $(\mathrm{p}<0.01)$, which was reversed by the Alendronate application $(\mathrm{p}<0.01)$. The BMP2 and ATF4 were mainly distributed in the cell boundaries. The Western blotting assays (Figure 5) of animal tissues also revealed that the relative protein expression of BMP2, EIF2AK3, EIF2A, ATF4 was decreased in the Model group, compared to the $\mathrm{NC}$
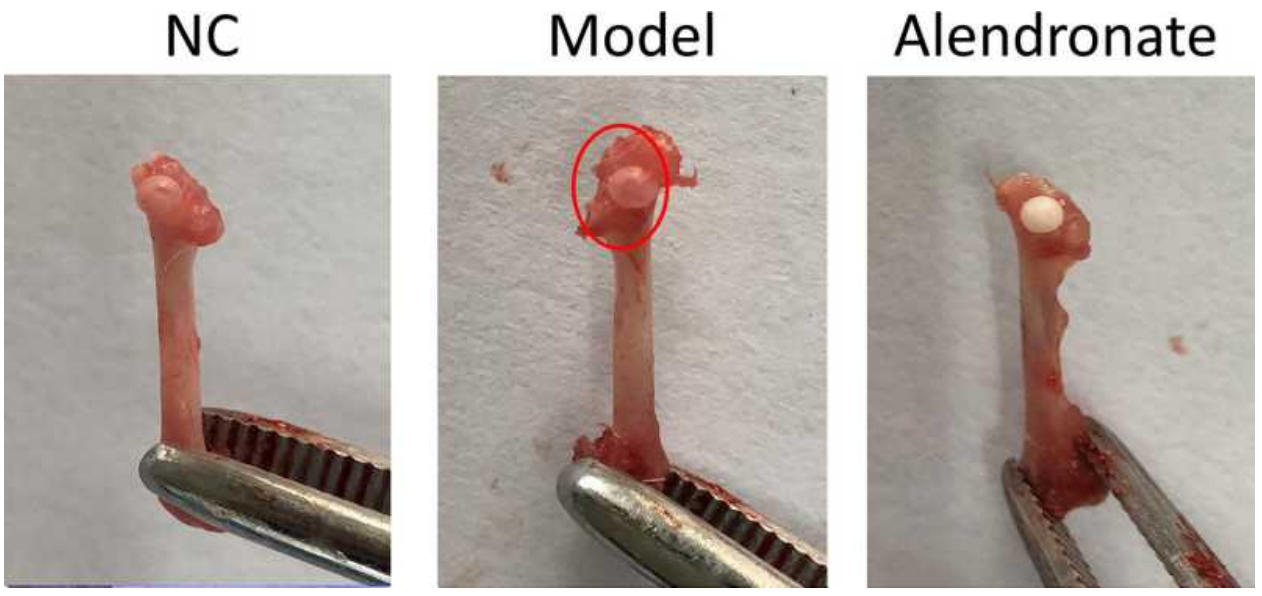

Figure 3 The morphological characteristics of osteonecrosis of femoral head. The red circle represented necrotic areas. 
A
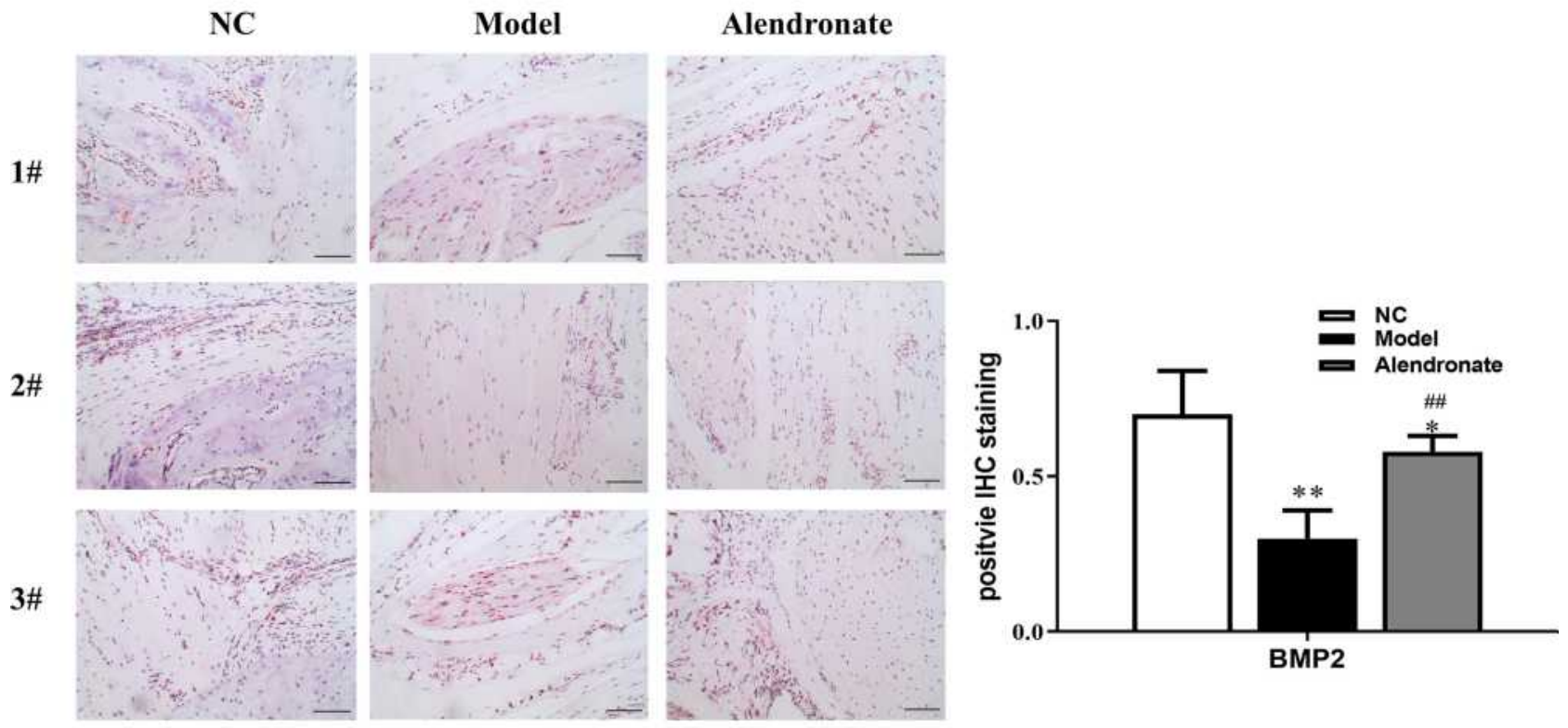

B
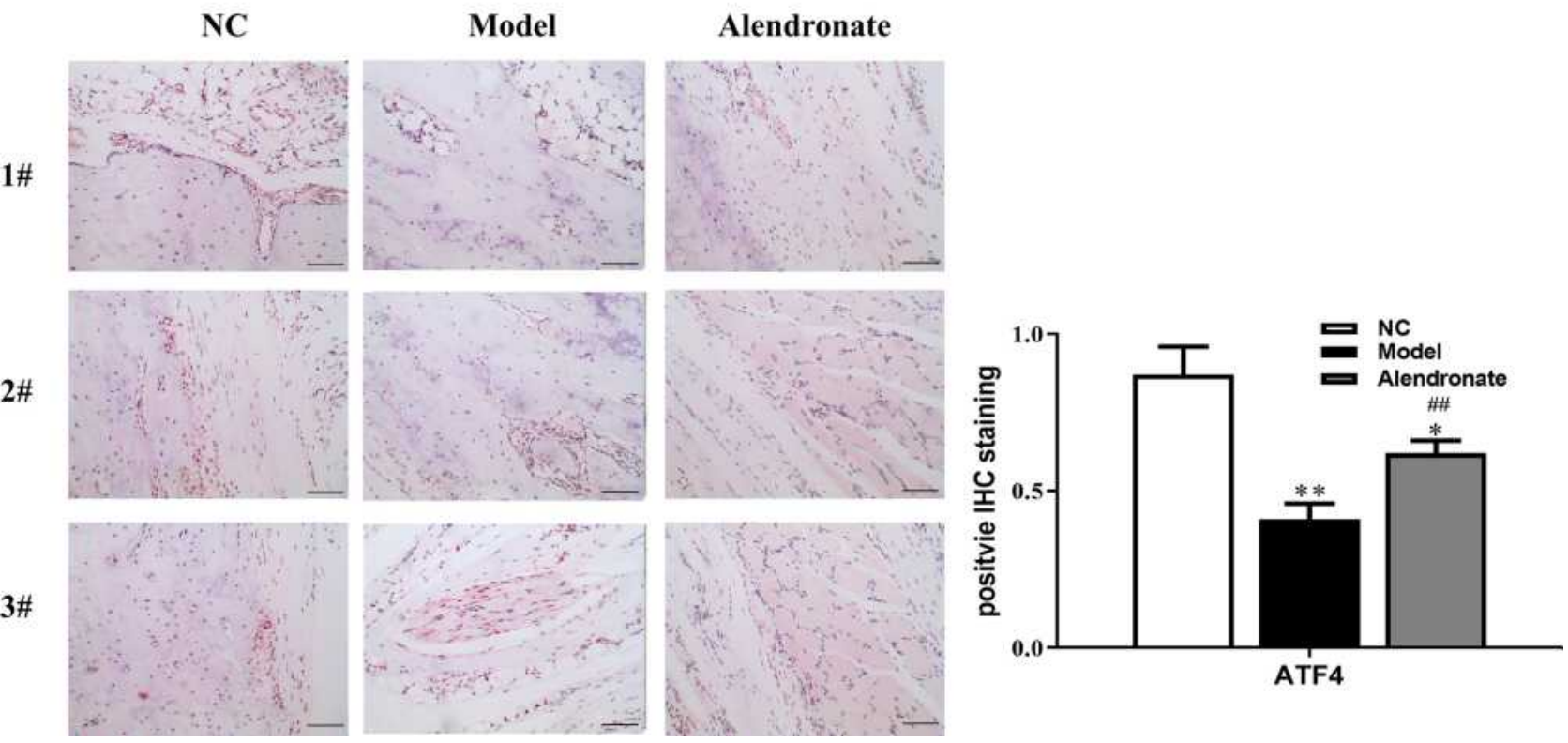

Figure 4 The expression of BMP2 (A) and ATF4 (B) in osteonecrosis and normal tissues detected by IHC. Bar $=50 \mu \mathrm{m}$. ${ }^{*}<0.05$, $*^{*}<<0.01$ compared with the NC group, $\#_{\mathrm{p}}<0.01$ compared with the Model group.

group $(\mathrm{p}<0.01)$, which was partially recovered by the Alendronate application $(\mathrm{p}<0.05)$.

\section{Discussion}

Recently, reports have demonstrated that the morbidity rate of ONFH shows an increasing trend year by year and it usually affects persons from 30 to 50 years old which is urgent to find an effective option to improve bone repair and inhibit articulus collapse. ${ }^{5,16}$ In particular, there are no effective treatment means except for hip replacement surgery in the late stage of ONFH and many patients require repeat surgery. Thus, various studies have been conducted to diagnose the disease in early stages. ${ }^{17,18}$

The pathogenesis of ONFH is complex and many of these etiologies are unclear, but eventually the changes 


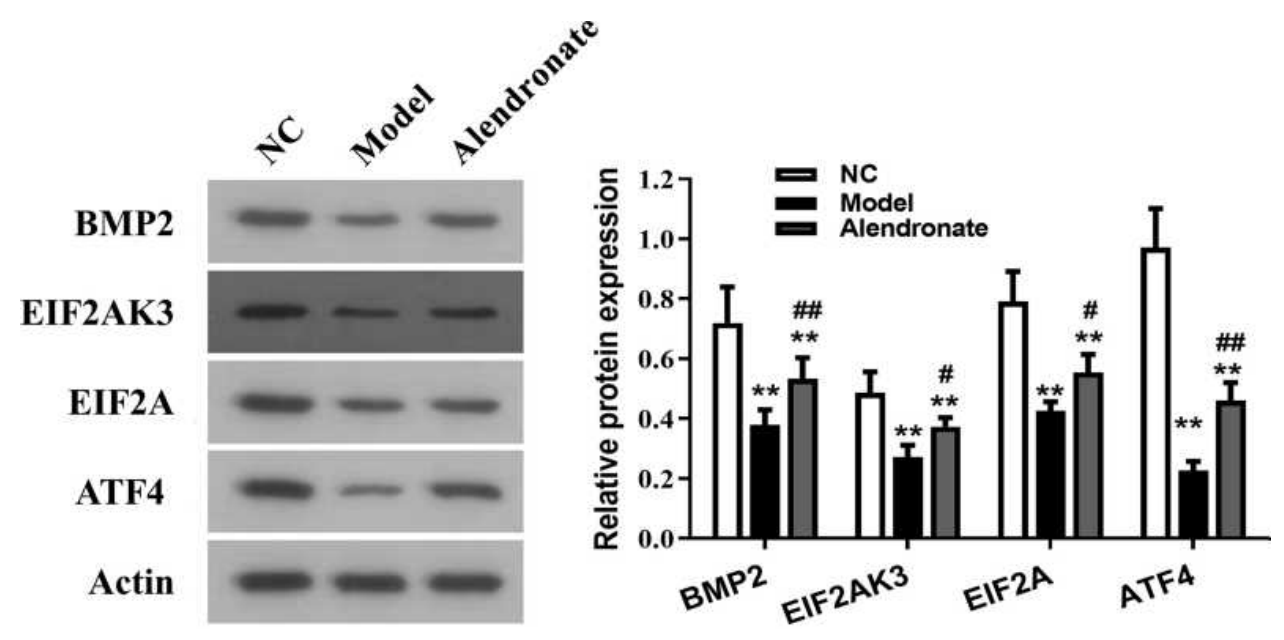

Figure 5 The relative protein expression of BMP2, ATF4 and TMEMII9 in the animal model detected using Western blotting. ** $<0.01$ compared with the NC group, ${ }_{\mathrm{p}}^{\#}<0.05,{ }^{\#} \mathrm{p}<0.01$ compared with the Model group.

in early stages, such as vascular damage, mechanical stresses and increased intraosseous pressure that occur result in failure to deliver nutrients to affected bone. BMP2 is one of the most important cytokines that plays several important roles in a variety of cellular functions, ranging from embryogenesis, cell growth and differentiation to bone development and fracture repair. ${ }^{19}$ Qian et al demonstrated that BMP2 enhanced bone regeneration by stimulating the Wnt signaling pathway. ${ }^{20}$ Tanaka et al showed that in myoblastic cells, BMP2 stimulates the phosphorylation of eIF $2 \alpha$, which acts downstream of EIF2AK3, and increases the osteoblast differentiating factor ATF4. ${ }^{7}$

As a kind of drug to reduce bone resorption by inhibiting osteoclast activity, Alendronate is generally used for treatment in the early stage of ONFH. Nishii et al found that Alendronate group showed a greater decrease of biochemical marker of bone resorption than biochemical marker of none formation and a lower frequency of collapse of the femoral head and reported less hip pain than the control group were shown as well. ${ }^{21}$ However, the effects of Alendronate on molecular pathways in the treatment of ONFH are rarely reported.

In this paper, therefore, the clinical samples of femoral head necrosis were collected to detect protein levels of BMP2, ATF4 and TMEM119. And the rat model of ONFH was established via the frozen liquid nitrogen, thus, the femoral head tissue could be collected to determine expression levels of BMP2, EIF2AK3, EIF2A and ATF4. The results showed that the expression level of BMP2, ATF4 and TMEM119 was significantly decreased in necrotic tissue of femoral head. Next, the pathological changes in the femoral head tissues were detected, and it was found that Alendronate could significantly improve the avascular necrosis of femoral head in ONFH rats. It was reported that ONFH was associated with synovitis, in which the proinflammatory cytokines that included TNF- $\alpha$ and IL-17 in necrotic lesions and synovium was significantly upregulated. ${ }^{22}$ Consistently, inflammatory muscle presented abnormal shape and color was observed in ONFH rats in the present study, which was also alleviated by Alendronate. According to the results of immunohistochemical analysis, Alendronate could remarkably increase the distribution and relative expression of BMP2 and ATF4, and the same tendency emerged in the expression of BMP2, EIF2AK3, EIF2A and ATF 4 by Western blot, indicating that the improvement of femoral head function in ONFH rats by Alendronate may be associated with the increased levels of osteoinductive factors in femoral head tissues. Vieira et al also found that Alendronate inhibited bone resorption, because it increased the expression of BMP2. ${ }^{23}$ Whether a single dose of Alendronate is associated with Alendronate resistance or decreased Alendronate susceptibility has not been demonstrated. Therefore, further studies should be conducted using multiple doses of Alendronate to screen out the most effective dose in multidisciplinary treatment of early-stage ONFH. Moreover, investigations on other biological changes such as ONFH-related advanced glycation end products are needed to comprehensively elucidate the effects of Alendronate. 


\section{Conclusion}

Alendronate application partially reversed the suppression of expression of BMP2, EIF2AK3, EIF2A, ATF4 caused by liquid nitrogen. Alendronate might be a promising drug for treating ONFH via targeting BMP2/EIF2AK3/EIF2A/ ATF4 pathway.

\section{Data Sharing Statement}

The data are free access to available upon request from the corresponding author.

\section{Ethics Approval and Consent to Participate}

The ethic approval was obtained from the Ethic Committee of The First Affiliated Hospital of Soochow University and written informed consent was obtained from all patients.

\section{Consent to Publish}

All of the authors have consented to publish this research.

\section{Acknowledgments}

We would like to acknowledge everyone for their helpful contributions on this paper. Ke Rong and Xiaoliu Li are co-first authors.

\section{Author Contributions}

All authors made a significant contribution to the work reported, whether that is in the conception, study design, execution, acquisition of data, analysis and interpretation, or in all these areas; took part in drafting, revising or critically reviewing the article; gave final approval of the version to be published; have agreed on the journal to which the article has been submitted; and agree to be accountable for all aspects of the work.

\section{Funding}

There is no funding to report.

\section{Disclosure}

All authors declare no conflicts of interest.

\section{References}

1. Zhou Z, Lin Y, Pan C, et al. IL-15 deficiency alleviates steroid-induced osteonecrosis of the femoral head by impact osteoclasts via RANKLRANK-OPG system. Immun Ageing. 2020;17(1):19. doi:10.1186/ s12979-020-00190-0
2. Chang C, Greenspan A, Gershwin ME. The pathogenesis, diagnosis and clinical manifestations of steroid-induced osteonecrosis. $J$ Autoimmun. 2020;110:102460. doi:10.1016/j.jaut.2020.102460

3. Ceponis P, Keilman C, Guerry C, Freiberger JJ. Hyperbaric oxygen therapy and osteonecrosis. Oral Dis. 2017;23(2):141-151. doi:10.1111/odi.12489

4. Murphey MD, Roberts CC, Bencardino JT, et al. ACR appropriateness criteria osteonecrosis of the hip. J Am Coll Radiol. 2016;13 (2):147-155. doi:10.1016/j.jacr.2015.10.033

5. Pan FY, Li ZM, Liu XW, et al. Effect of strontium ranelate on rabbits with steroid-induced osteonecrosis of femoral head through TGF- $\beta 1 /$ BMP2 pathway. Eur Rev Med Pharmacol Sci. 2020;24(3):10001006. doi:10.26355/eurrev_202002_20150

6. Bragdon B, Moseychuk O, Saldanha S, King D, Julian J, Nohe A. Bone morphogenetic proteins: a critical review. Cell Signal. 2011;23 (4):609-620. doi:10.1016/j.cellsig.2010.10.003

7. Tanaka K, Kaji H, Yamaguchi T, et al. Involvement of the osteoinductive factors, Tmem119 and BMP-2, and the ER stress response PERK-eIF2 $\alpha$-ATF4 pathway in the commitment of myoblastic into osteoblastic cells. Calcif Tissue Int. 2014;94(4):454-464. doi:10.1007/s00223-013-9828-1

8. Hisa I, Inoue Y, Hendy GN, et al. Parathyroid hormone-responsive Smad3-related factor, Tmem119, promotes osteoblast differentiation and interacts with the bone morphogenetic protein-Runx2 pathway. $J$ Biol Chem. 2011;286(11):9787-9796. doi:10.1074/jbc.M110.179127

9. Satoh J, Kino Y, Asahina N, et al. TMEM119 marks a subset of microglia in the human brain. Neuropathology. 2016;36(1):39-49. doi:10.1111/neup. 12235

10. Acar N, Balkarli H, Soyuncu Y, et al. The determination of apoptosis rates on articular cartilages of ovariectomized rats with and without alendronate treatment. Histol Histopathol. 2016;31(6):635-645. doi:10.14670/HH-11-701

11. Wang YK, Zhang YM, Qin SQ, et al. Effects of alendronate for treatment of glucocorticoid-induced osteoporosis: a meta-analysis of randomized controlled trials. Medicine (Baltimore). 2018;97(42): e12691. doi:10.1097/MD.0000000000012691

12. Yin Y, Ding L, Hou Y, et al. Upregulating MicroRNA-410 or downregulating Wnt-11 increases osteoblasts and reduces osteoclasts to alleviate osteonecrosis of the femoral head. Nanoscale Res Lett. 2019;14(1):383. doi:10.1186/s11671-019-3221-6

13. Matsutani S, Shibutani M, Maeda K, et al. Tumor-infiltrating immune cells in H\&E-stained sections of colorectal cancer tissue as a reasonable immunological biomarker. Anticancer Res. 2018;38(12):67216727. doi:10.21873/anticanres.13041

14. Kurien BT, Scofield RH. Western blotting: an introduction. Methods Mol Biol. 2015;1312:17-30.

15. Zhou Y, Zhou Y, Wang K, et al. ROCK2 confers acquired gemcitabine resistance in pancreatic cancer cells by upregulating transcription factor ZEB1. Cancers (Basel). 2019;11(12):1881. doi:10.3390/ cancers 11121881

16. Wen Z, Lin Z, Yan W, Zhang J. Influence of cigarette smoking on osteonecrosis of the femoral head (ONFH): a systematic review and meta-analysis. Hip Int. 2017;27(5):425-435. doi:10.5301/hipint.5000516

17. Popere S, Shinde SS, Patel R, Kulkarni A. A cross sectional study of outcomes of muscle pedicle grafting in neck of femur fractures and avascular necrosis of femoral head. Injury. 2020;51(7):1622-1625. doi:10.1016/j.injury.2020.04.026

18. Zhun W, Donghai L, Zhouyuan Y, Haiyan Z, Pengde K. Efficiency of cell therapy to GC-Induced ONFH:BMSCs with Dkk-1 interference is not superior to unmodified BMSCs. Stem Cells Int. 2018;2018:1340252. doi:10.1155/2018/1340252

19. Dudakovic A, Samsonraj RM, Paradise CR, et al. Inhibition of the epigenetic suppressor EZH2 primes osteogenic differentiation mediated by BMP2. J Biol Chem. 2020;295(23):7877-7893. doi:10.1074/jbc.RA119.011685 
20. Qian C, Zhu C, Yu W, Jiang X, Zhang F, Sun J. Bone morphogenetic protein 2 promotes osteogenesis of bone marrow stromal cells in type 2 diabetic rats via the Wnt signaling pathway. Int J Biochem Cell Biol. 2016;80:143-153. doi:10.1016/j.biocel.2016.09.025

21. Nishii T, Sugano N, Miki H, Hashimoto J, Yoshikawa H. Does alendronate prevent collapse in osteonecrosis of the femoral head? Clin Orthop Relat Res. 2006;443:273-279. doi:10.1097/01. blo.0000194078.32776.31
22. Han J, Gao F, Li Y, et al. The use of platelet-rich plasma for the treatment of osteonecrosis of the femoral head: a systematic review. Biomed Res Int. 2020;2020:2642439. doi:10.1155/2020/2642439

23. Vieira JS, Cunha EJ, de Souza JF, et al. Alendronate induces postnatal maxillary bone growth by stimulating intramembranous ossification and preventing premature cartilage mineralization in the midpalatal suture of newborn rats. Int J Oral Maxillofac Surg. 2019;48(11):1494-1503. doi:10.1016/j.ijom.2019.04.002

\section{Publish your work in this journal}

Drug Design, Development and Therapy is an international, peerreviewed open-access journal that spans the spectrum of drug design and development through to clinical applications. Clinical outcomes, patient safety, and programs for the development and effective, safe, and sustained use of medicines are a feature of the journal, which has also been accepted for indexing on PubMed Central. The manuscrip management system is completely online and includes a very quick and fair peer-review system, which is all easy to use. Visit http://www. dovepress.com/testimonials.php to read real quotes from published authors. 